\title{
In silico and in vitro evaluation of brain penetration properties of selected nootropic agents
}

\author{
Ahmed Alsarrani**,1 \& Paul V Kaplita*,1 \\ ${ }^{1}$ Department of Pharmaceutical Sciences, School of Pharmacy, MCPHS University, 19 Foster Street, Worcester, MA 01608, USA \\ *Author for correspondence: Tel.. +1 508373 5614; Fax: +1 508890 5618; paul.kaplita@mcphs.edu \\ **Author for correspondence: Tel.: +1 954262 1112; aa2805@mynsu.nova.edu
}

\begin{abstract}
Aim: Predictive (in silico) data suggested that nootropic supplements may penetrate the blood-brain barrier (BBB). We evaluated, in vitro, the ability of nootropics to enter the brain based on the high throughput screening (HTS) measurement of interactions with the P-gp efflux transporter and physicochemical properties and correlated these data with the in silico predictions. Methods \& results: The software predicted that piracetam, docosahexaenoic acid (DHA), amantadine and thioflavin-T can best penetrate the BBB. The lipophilicity of these compounds may be predicted by measuring the critical micelle concentration (CMC). DHA and verapamil demonstrated high lipophilicity. DHA, verapamil and phosphatidylserine (PS) may be good substrates of the P-gp transporter. Conclusion: Permeability of nootropics may be successfully predicted by high throughput screening-lead optimization assay technologies.
\end{abstract}

Lay abstract: There is high interest among people for using nutritional supplements and drugs to enhance mental performance (i.e., nootropics). Although the mechanism of action of these supplements is not precisely known, several studies suggest that they may enhance the vitality of neurons and connections between them. More clinical research is needed with both healthy and diseased adult subjects and there is very little preclinical, laboratory research available on nootropics. There is a particular lack of research on the chemical properties that would make them capable of entering the brain. Our work evaluates computational and experimental technologies that may predict these properties.

First draft submitted: 8 February 2019; Accepted for publication: 30 April 2019; Published online: 10 June 2019

Keywords: blood-brain barrier $\bullet$ in silico to in vitro extrapolation • lipophilicity $\bullet$ nootropic $\bullet$ P-glycoprotein

Cognitive skills are vital mental functions that include memory, learning and creativity [1]. Numerous drugs, supplements and even healthy lifestyle habits have been proposed to strengthen cognitive skills $[2,3]$. The term nootropic was originally limited to the drug piracetam, which showed some cognitive enhancement effects $[4,5]$. Eventually the definition was expanded to cover any drug or dietary supplement that may enhance cognitive functions. In 2015, the sales of nootropics worldwide were around US $\$ 1$ billion [6]. As they exhibit minimal side effects (e.g., sleep disturbance, headache, insomnia and anxiety) [5], they have been widely marketed as over-thecounter drugs in a single or a mixed form [7]. Many healthy elderly adults and adolescents are using them to improve their cognitive skills. The use of supplements by healthy people seeking a neurological effect has been a controversial subject among neuroscientists and psychiatrists [8]. In particular, this group of scientists does not recommend the use of these supplements by children [9]. However, using these compounds on a regular basis has been claimed to protect the brain and promote cognitive functions [2]. More clinical research is needed with both healthy and diseased adult subjects, as well as preclinical, laboratory research into the actions of nootropics [10]. Racetam-related nootropics and neuro-nutritional supplements are common classes that may be considered as cognitive enhancers.

Racetam-related drugs are structurally similar pyrrolidone derivatives and are the first class to be considered nootropics [11]. Laboratory studies suggest that these drugs may improve brain function by increasing neuronal calcium influx by acting as positive allosteric modulators of $\alpha$-amino-3-hydroxy-5-methyl-4-isoxazolepropionic acid (AMPA) ionotropic glutamate subtype receptors [12]. Activating $\alpha$-amino-3-hydroxy-5-methyl-4-isoxazolepropionic 
acid receptors at the allosteric site may produce enhancement without causing neurotoxicity [13]. Some studies also suggest that racetams may increase acetylcholine (ACh) production [11]. These drugs are regarded as safe and there are a few documented adverse effects of racetams, such as anxiety and sleeplessness [14]. Piracetam may protect the brain from oxidative stress and excitotoxicity, and preserve the integrity and structure of neurons [14-17]. The analog, aniracetam, has been shown to be less effective in improving the cognitive functions [18]. Oxiracetam, with a hydroxyl group substitution in its oxo-pyrrolidone moiety, has better bioavailability [19]. Pramiracetam also enhanced memory in a placebo-controlled study of pramiracetam in young males with memory and cognitive problems [20]. Phenylpiracetam has also been reported to have better bioavailability than piracetam [5].

Many nutritional materials have been used to support brain functions [21] and enhance cognitive skills by restoring the loss of membrane lipid composition [22] or mimicking excitatory neurotransmitter activity [23]. It has been suggested that these materials are essential substances that make neurons more vital and active by supporting the strength of synaptic plasticity, either by involvement in neurotransmitter synthesis or supporting the structure of neurons. Docosahexaenoic acid (DHA), phosphatidylserine (PS) and L-theanine are widely used examples for this class. DHA is an endogenous omega-3 fatty acid found in neurons, it is one of the dietary fatty acids that have been used to support brain function [24]. DHA is a cell membrane phospholipid that is important for neuron cell structure [25] and its ingestion has been suggested as a neuro-nutrient supplement [26-28]; however, some reports revealed a lack of effectiveness of DHA on cognition improvement [29,30]. PS, a cell phospholipid, has a role in cell cycle signaling [31], with several studies having shown that PS can enhance cognitive skills in elderly people [32]. It can be extracted from bovine brain cortex and also may be found in soy [33]. Some reports showed that PS from brain cortex was able to improve acetylcholine release [32] and PS supplementation may enhance the cognition by supporting cell membrane composition [34]. L-theanine is an amino acid that resembles the chemical structure of the excitatory neurotransmitter glutamate. Some reports show that it may partially bind to glutamate receptors [35]. It has been approved as a mental enhancing agent in many countries such as Japan [23] and is naturally found in green tea. Some reports show that L-theanine might protect against neuronal death [36] though there is a lack of research on the effects of L-theanine in humans.

There is a particular lack of research on the pharmacokinetic behavior of most nootropic supplements. In this study, computational, biochemical and physicochemical laboratory data have been collected to test the following hypothesis: selected nootropics or neuro-nutrients have physico-chemical properties that would make them capable of entering the brain by crossing the blood-brain barrier (BBB). The goal of this study is to estimate, both in silico and in vitro, the ability of these chosen nootropics to move from the bloodstream into the brain in vivo by penetrating the BBB. This estimation is based on comparing some physicochemical parameters (such as lipophilicity) and the biochemical interaction with a specific membrane transporter such as P-gp, with corresponding in silico predictive data.

\section{Materials \& methods Materials}

The nootropic drugs used in the study were piracetam, oxiracetam and aniracetam. DHA, PS and L-theanine were used as neuro-nutrient supplements. Verapamil was used as a P-gp positive control and amantadine as a P-gp no-effect control. Thioflavin- $T$, a molecule known to cross the BBB and used as a dye for amyloid- $\beta$ detection, was tested as a proof-of-principle (i.e., entry into brain) control. All compounds were purchased from Sigma Aldrich ${ }^{\mathrm{TM}}$ (MO, USA).

\section{Physicochemical parameters prediction software}

Optibrium StarDrop ${ }^{T M}$ software (Cambridge, UK) was used to calculate the in silico physicochemical parameters for each given chemical structure. There are several key parameters for BBB penetration that can be predicted by this software, such as $\log \mathrm{S}, \log \mathrm{P}, \log \mathrm{D}, \mathrm{BBB}$ partition factor (an ensemble) and P-gp substrate factor (an ensemble). These data allow prioritization of these compounds for further assessment in the study. A license for the Optibrium StarDrop ${ }^{\mathrm{TM}}$ software suite is available within the Department of Pharmaceutical Sciences.

\section{Surface tension measurement}

A Kibron (Helsinki, Finland) microtensiometer used the Wilhelmy plate method to measure the potency of selected nootropics to alter the air:water surface tension of water droplets (critical micelle concentration, or CMC). This technique has been documented as a model that accurately predicts passive in vivo permeability of drugs through 
the BBB. Simply, $350 \mu \mathrm{l}$ of distilled water was added to the well at ambient temperature and then a needle was immersed in the water droplet. The surface tension of the water is measured after $30 \mathrm{~s}$. Then, $150 \mu \mathrm{l}$ of a particular concentration of a compound was added. The surface tension was then measured after 20 min to allow for the equilibration of the compound within the droplet. The change in the surface tension versus test compound (TC) concentration relationship was visually demarcated as the potency of the compound to change the air:water surface tension of water droplets (CMC). This analysis accurately models passive in vivo permeability of drugs through the BBB [37]. The zwitterionic detergent, 3-[(3-cholamidopropyl)-dimethylammonio]-1-propanesulfonate (CHAPS) was used as a positive control for surface tension measurement [38].

\section{P-gp ATPase assay}

The P-gp-Glo ${ }^{T M}$ Assay System (Promega Corporation, WI, USA) was used to observe the effects of the compounds on the activity of P-gp ATPase. The assay determines the P-gp ATPase activity of a compound through the ATP dependence of the light-generating reaction of firefly luciferase. A higher P-gp ATPase activity will consume more ATP, which eventually results in a low light output. P-gp membranes were prepared from human P-gp overexpression in insect cells using a baculovirus system and a membrane fraction is prepared. The experimental conditions followed the manufacturer's specifications [39]. Several concentrations of TCs were pre-incubated with recombinant human P-gp membranes in assay buffer at $37^{\circ} \mathrm{C}$ for $5 \mathrm{~min}$. The ATP hydrolysis reaction was initiated by adding $10 \mu \mathrm{l}$ ATP, followed by incubation at $37^{\circ} \mathrm{C}$ for $60 \mathrm{~min}$. During the incubation time, some of the ATP was consumed by P-gp. The reaction was terminated by adding an ATP detection reagent, followed by incubation at room temperature for $20 \mathrm{~min}$ to allow for the luminescent signal to develop. Subsequently, luminescence was determined using a Multi-Mode Microplate Reader Synergy ${ }^{T M}$ HT (BioTek Instruments, VT, USA). ATP consumption by P-gp was observed as a decrease in luminescence compared with the nontreated control. TCs that stimulate the P-gp ATPase activity will reduce luminescence compared with an untreated control. In this way substrates for transport by P-gp are identified as stimulators of the ATPase activity. Inhibitors of the ATPase activity are identified as compounds that reduce ATPase activity, and in turn, elevate the luminescence that can be observed with basal activity or drug-stimulated activity. $\mathrm{Na}_{3} \mathrm{VO}_{4}$ is an inhibitor of membrane-bound ATPases, this treatment served as a control where no P-gp-dependent ATP consumption occurs. In the untreated sample, P-gp is anticipated to consume ATP at a basal rate, so this rate was compared with the $\mathrm{Na}_{3} \mathrm{VO}_{4}$-treated sample and verapamil-treated samples. The untreated sample showed a decrease in luminescence compared with the $\mathrm{Na}_{3} \mathrm{VO}_{4}$-treated sample, and that difference in relative light units of basal $\left(\triangle \mathrm{RLU}_{\text {basal }}\right)$ reflects the basal P-gp ATPase activity. Verapamil caused a more considerable decrease in luminescence compared with basal levels, and that reduction $\left(\triangle R L U_{T C}\right)$ reflects the verapamil-stimulated ATPase activity.

\section{Statistical analysis}

The data were analyzed using GraphPad Prism version 5.00 for Windows (GraphPad Software, CA, USA) and differences between groups were analyzed using the unpaired t-test. Results are presented as mean \pm standard error of the mean (SEM). For the P-gp assays, experiments were repeated three-times, and the mean ratio \pm SEM was presented. The $\mathrm{p}<0.005$, with one-way ANOVA analysis (*treatment vs basal).

\section{Results}

\section{Physicochemical parameters prediction}

Several representative nootropics, the P-gp no-effect control (amantadine), the positive P-gp control (verapamil) and a proof-of-principle control (thioflavin-T) were evaluated with Optibrium Stardrop ${ }^{T M}$, a validated, proprietary software. The data are summarized in Table 1 .

Passive diffusion into the brain may be achieved by high lipid solubility (i.e., large $\log$ P, $\log$ D), low hydrogen bonding and molecular mass (i.e., small molecular weight [MW]). Based on the computational data from Table 1, the nootropic or control compounds that can best penetrate the BBB are piracetam, aniracetam, DHA, thioflavin-T and amantadine. Aniracetam, DHA, thioflavin-T, amantadine and verapamil would possess high lipophilicity (log P); while PS, piracetam, oxiracetam and L-theanine would be poorly lipid soluble. PS, L-theanine, oxiracetam and verapamil have the most H-bond capacity. Only PS and verapamil would be expected to interact with P-gp while amantadine has no effect on P-gp. 


\begin{tabular}{|c|c|c|c|c|c|c|c|c|c|c|}
\hline Compound & $\begin{array}{l}\log 5 @ \\
\text { pH7.4 }\end{array}$ & $\log P$ & $\log D$ & BBB category & $\begin{array}{l}\text { P-gp } \\
\text { category }\end{array}$ & HBD & HBA & TPSA & MW & $\begin{array}{l}\text { Rotatable } \\
\text { bonds }\end{array}$ \\
\hline Piracetam & 6.092 & -1.54 & -1.54 & + & No & 1 & 4 & 63.4 & 142.2 & 2 \\
\hline Oxiracetam & 6.233 & -1.914 & -1.914 & - & No & 2 & 5 & 83.63 & 158.2 & 2 \\
\hline Aniracetam & 3.94 & 1.208 & 1.208 & + & No & 0 & 4 & 46.61 & 219.2 & 3 \\
\hline L-Theanine & 4.138 & -2.934 & -2.796 & - & No & 3 & 5 & 92.42 & 174.2 & 6 \\
\hline DHA & 1.779 & 4.952 & 4.952 & + & No & 0 & 2 & 26.3 & 356.5 & 16 \\
\hline Phosphatidylserine & 4.733 & -1.886 & -1.839 & - & Yes & 3 & 11 & 171.7 & 385.3 & 15 \\
\hline Amantadine & 2.737 & 2.44 & -0.2159 & + & No & 1 & 1 & 26.02 & 151.2 & 0 \\
\hline Thioflavin- $T$ & 2.136 & 4.494 & 2.056 & + & No & 0 & 2 & 7.12 & 283.4 & 2 \\
\hline Verapamil & 2.941 & 3.79 & 1.91 & - & Yes & 0 & 6 & 63.95 & 454.6 & 14 \\
\hline \multicolumn{11}{|c|}{$\begin{array}{l}\text { Log S @ pH 7.4 is the predicted logarithm of the apparent solubility at pH 7.4, } \mathrm{S} \text { in } \mu \mathrm{M} \text {, for ionized compounds. Log P is the predicted logarithm of the octanol/water partition } \\
\text { coefficient for neutral compounds. Log D is the predicted logarithm of the octanol/water partition coefficient for ionized compounds at a fixed pH of } 7.4 \text {. For BBB category (an } \\
\text { ensemble prediction), a classification of ' ' }+ \text { ' indicates that BBB penetration is usually present unless the compound is a substrate for active efflux transporters such as P-gp, while } \\
\text { classification of '-' indicates that there is likely to be little or no BBB penetration. P-gp category is an ensemble prediction for P-gp efflux transport. It predicts a classification of 'yes' } \\
\text { for substrates and 'no' for non-substrates. HBD is the number of HBDs in the TC. HBA is the number of HBAs in the TC. MW is the MW of the TC. Rotatable bonds are the number } \\
\text { of rotatable bonds which are the number of bonds that allow free rotation around themselves. } \\
\text { BBB: Blood-brain barrier; DHA: Docosahexaenoic acid; HBA: Hydrogen-bond acceptor; HBD: Hydrogen-bond donor; MW: Molecular weight; P-gp: P-glycoprotein; TC: Test compound; } \\
\text { TPSA: Topological polar surface area. }\end{array}$} \\
\hline
\end{tabular}

\begin{tabular}{|l|l|}
\hline Table 2. Critical micelle concentration values (average of $n=3-5$ experiments) for nootropic and control compounds. \\
\begin{tabular}{|l|l|}
\hline CMC $(\mu \mathrm{M})$ & 1000 \\
Piracetam & 1000 \\
\hline Oxiracetam & 1000 \\
\hline Aniracetam & 1000 \\
\hline Phosphatidylserine & 100 \\
\hline DHA & 10,000 \\
\hline CHAPS & 10 \\
\hline Verapamil & 1000 \\
\hline Amantadine & 1000 \\
\hline Thioflavin- & ND \\
\hline L-theanine & \\
\hline Results were presented as average with \pm standard error of the mean. \\
CHAPS: 3 -[(3-cholamidopropyl)-dimethylammonio]-1-propanesulfonate; CMC: Critical micelle concentration; DHA: Docosahexaenoic acid; ND: Could not be determined.
\end{tabular} \\
\hline
\end{tabular}

\section{Surface tension measurement}

Compounds with hydrophobic (lipid soluble) moieties aggregate at the air/water interfaces of water droplets when they reach their solubility limits, forming micelles. We were able to observe this using the Wilhelmy plate method. This solubility limit or CMC can be observed visually at the deflection point in the concentration versus surface tension curve (Figures 1-4). This reflects a transition above which the concentration of free hydrophobic molecules remains constant, resulting in a plateau in the curve.

As shown above in Table 2, the CMC values of verapamil and DHA are low and so may indicate the high lipophilic properties of these compounds. The CMC value of the zwitterionic detergent control CHAPS was consistent with published literature results [38] and was more significant than the other compounds tested in these experiments. Piracetam, aniracetam, oxiracetam, PS and thioflavin-T and amantadine show quite high CMC as they induce micelle formation around $1000 \mu \mathrm{M}$. Data were unclear for L-theanine.

\section{P-gp ATPase assay}

P-gp is an ATP-dependent efflux pump for many hydrophobic (lipid-soluble) compounds. The interaction of compounds with P-gp may be measured by observing their effect on the rate of P-gp ATP hydrolysis. For example, compounds that do not interact with P-gp will not affect basal ATPase activity, compounds that are substrates for P-gp transport will stimulate basal ATPase activity and compounds that inhibit P-gp will inhibit basal and stimulated ATPase activity. 


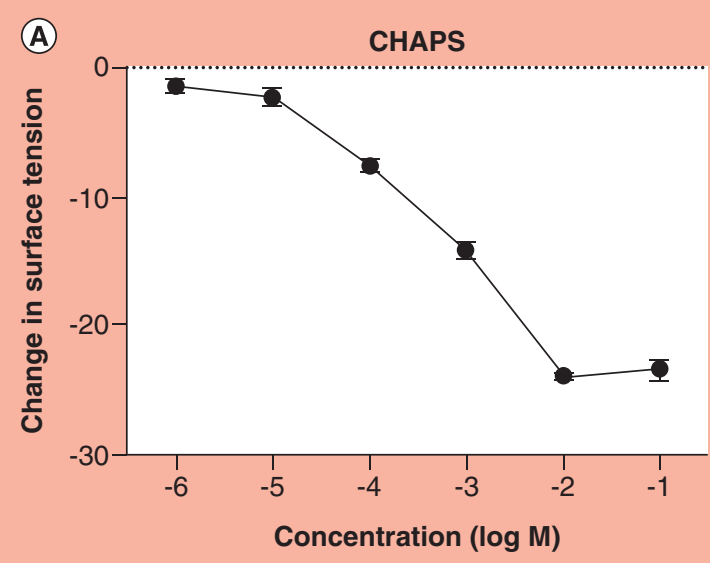

(C)

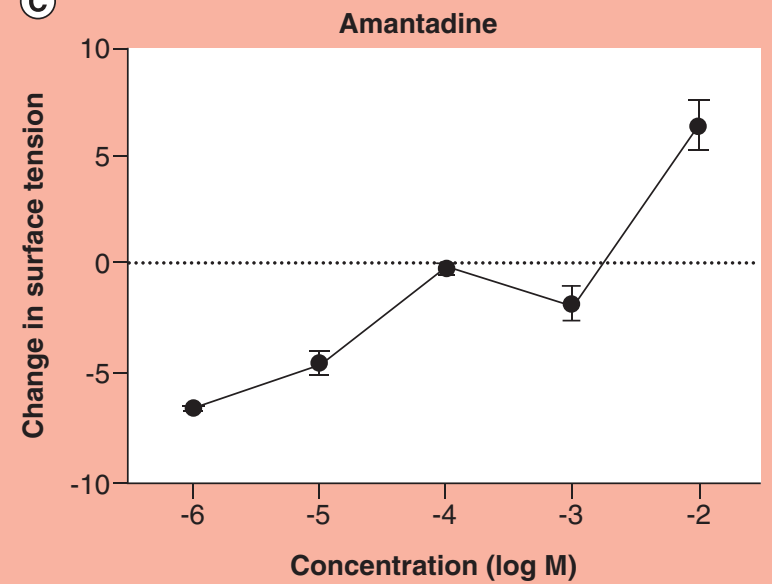

(B)

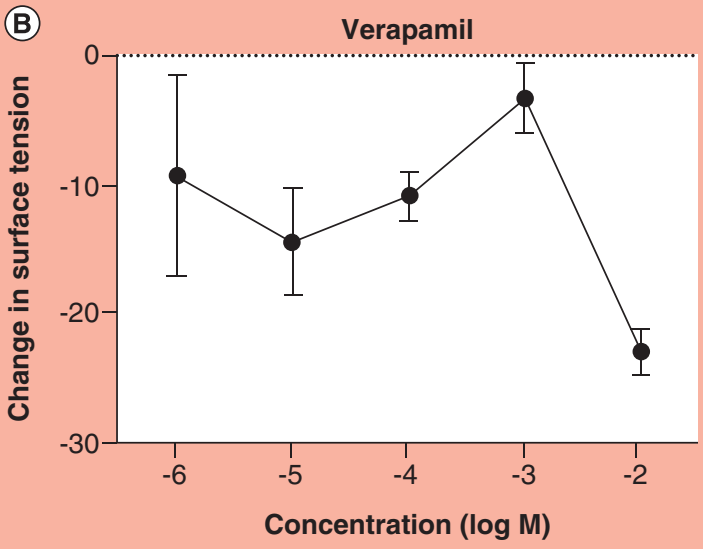

(D)

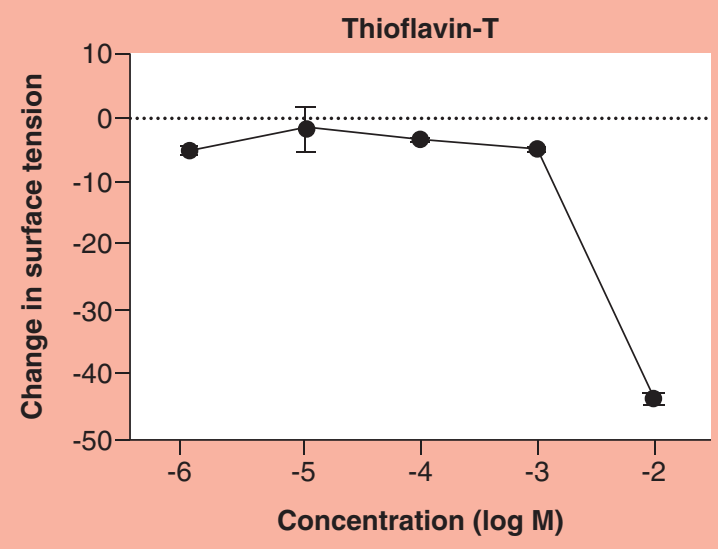

Figure 1. Critical micelle concentration curves for control compounds. (A) CHAPS, (B) verapamil, (C) amantadine and (D) Thioflavin-T. Results were presented as mean with \pm standard error of the mean. The critical micelle concentration was qualitatively determined from the visual inflection point in the curves.

CHAPS: 3-[(3-cholamidopropyl)-dimethylammonio)-1-propanesulfonate.

Table 3. Smallest concentration values (average of $n=3-10$ experiments) for nootropic and control compounds that stimulate basal P-glycoprotein-associated ATPase activity by threefold.

\begin{tabular}{|ll|}
\hline Compound & P-gp ATPase $(\mu \mathrm{M})$ \\
\hline Piracetam & NS \\
\hline Oxiracetam & NS \\
\hline DHiracetam & NS \\
\hline L-Theanine & 1 \\
\hline Phosphatidylserine & NS \\
\hline Amantadine & 30 \\
\hline Verapamil & NS \\
\hline Thioflavin-T & 50 \\
\hline DHA: Docosahexaenoic acid; NS: Not significant. & NS \\
\hline
\end{tabular}

The experimental observations (Table 3; Figures 5-9) suggest that PS and verapamil are the most effective stimulators of P-gp ATPase, while piracetam, amantadine, thioflavin-T, oxiracetam and L-theanine exhibit little 

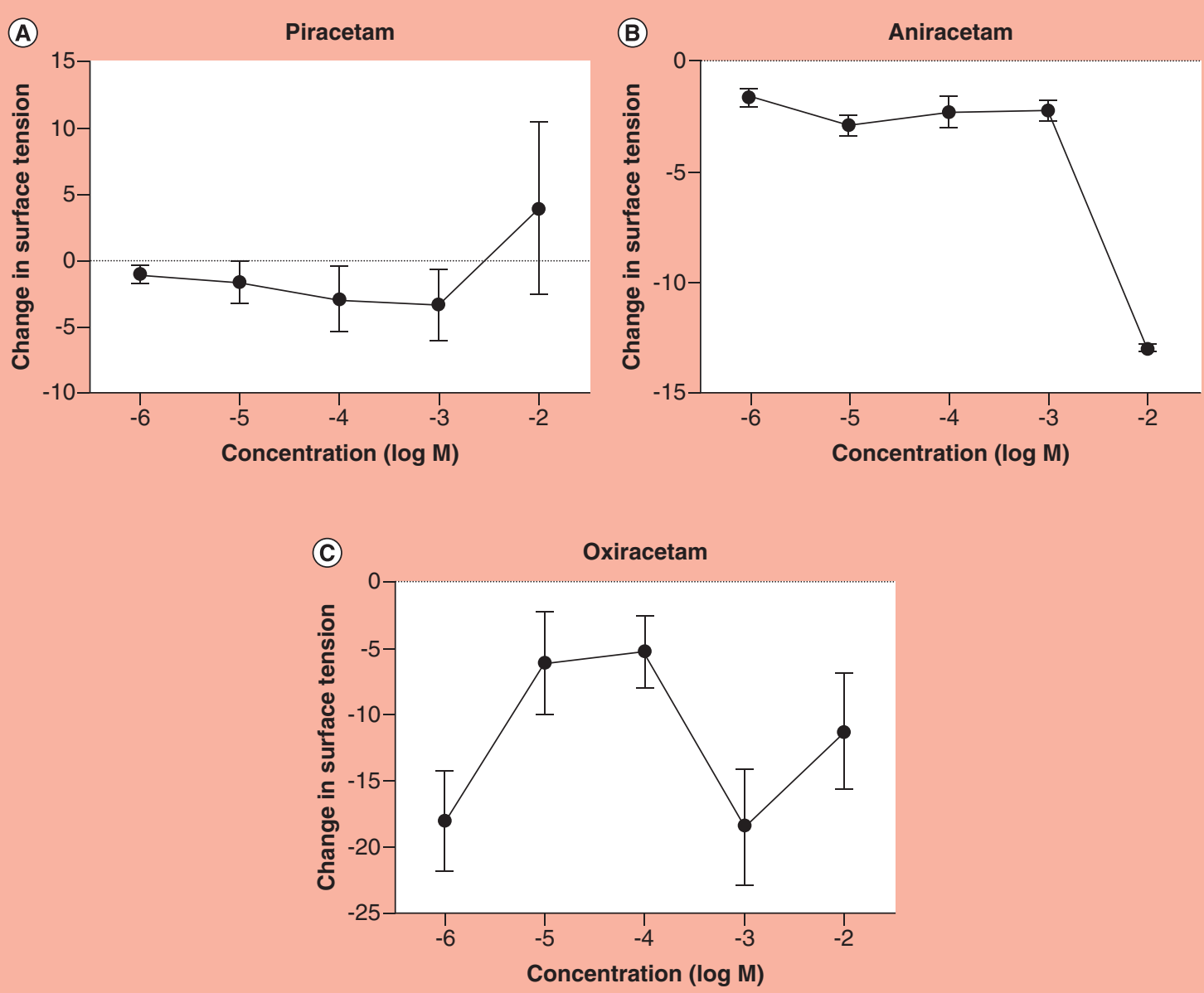

Figure 2. Critical micelle concentration curves for racetam-related nootropic compounds. (A) Piracetam, (B) aniracetam and (C) oxiracetam. Results were presented as mean with \pm standard error of the mean. The critical micelle concentration was qualitatively determined from the visual inflection point in the curves.

to no effect. These data are consistent with the in silico data. At $1 \mu \mathrm{M}$, DHA appears to stimulate P-gp ATPase activity but then at higher concentrations, it appears to inhibit or interfere with P-gp ATPase activity.

\section{Discussion}

There is very little preclinical, laboratory research available on the pharmacokinetics and pharmacodynamics of nootropic drugs [5,11-20] and supplements [21-23]. In particular, it is well known that the BBB is a significant challenge for the entry of drugs and supplements into the brain [40]. The BBB has a highly complex, semipermeable membrane structure that allows the passage into the brain of substances only with specific physicochemical properties [41,42]. It hinders the passage of drugs to reach the therapeutic levels in the brain. Hence, the objective of this study is to estimate and compare the in silico and in vitro ability of selected nootropics and neuro-nutrients to potentially cross the $\mathrm{BBB}$ in vivo.

$\mathrm{BBB}$ penetration is one of the major obstacles to the development of CNS drugs. It is considered to be one of the critical pharmacokinetic/pharmacodynamic factors that may affect the efficacy of many CNS drugs. Its structural complexity limits molecules with only highly specific characteristics to move passively through the BBB. High lipophilicity, low hydrogen bonding capacity and low molecular weight [43] are major favorable features for passive diffusion. This has been broadly summarized for small molecules by Lipinski's Rules of Five [44], which suggested five general chemical rules for membrane penetration:

- Molecular weight is less than 500 Daltons. 

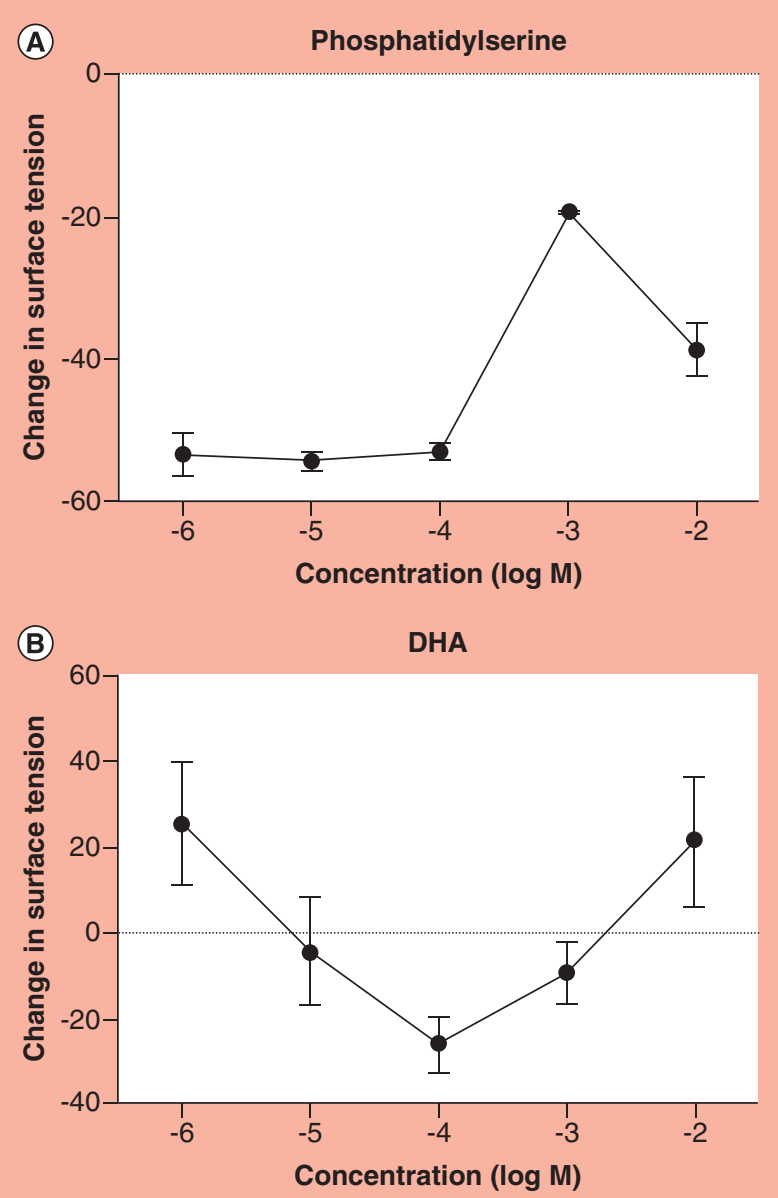

Figure 3. Critical micelle concentration curves for neuro-nutrient supplements. (A) Phosphatidylserine and (B) DHA. Results were presented as mean with \pm standard error of the mean. The critical micelle concentration was qualitatively determined from the visual inflection point in the curves.

DHA: Docosahexaenoic acid.

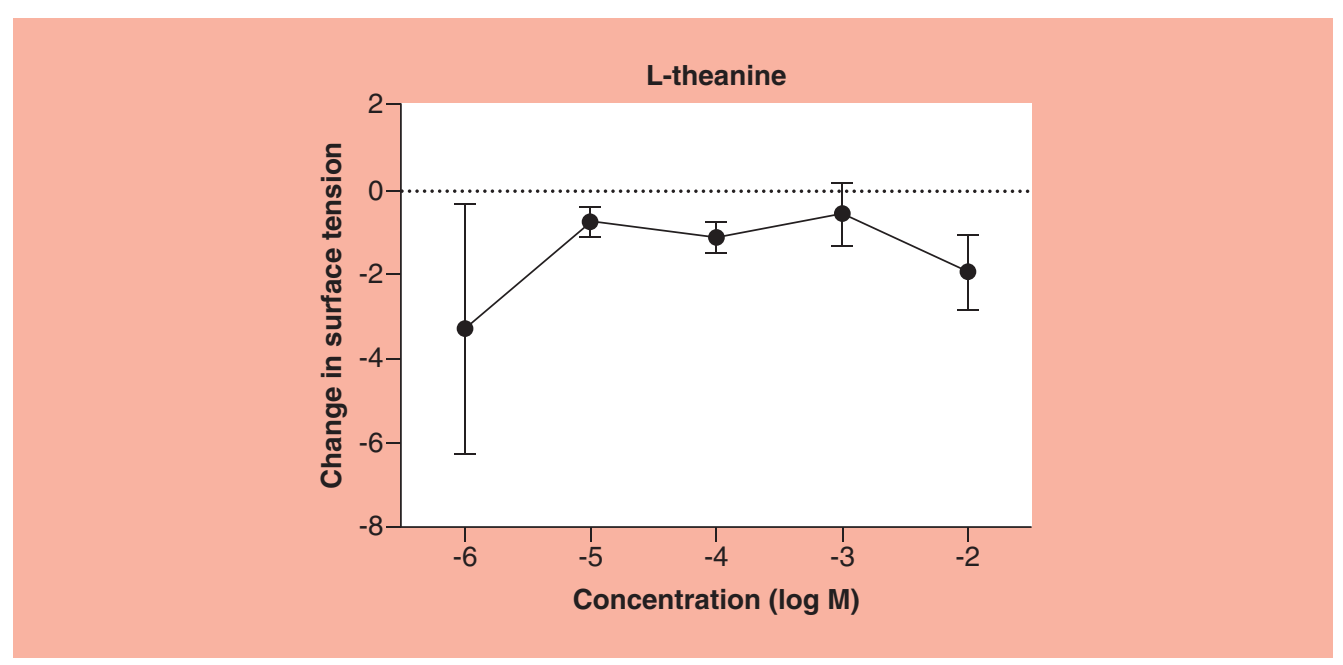

Figure 4. Critical micelle concentration curve for L-theanine. Results were presented as mean with \pm standard error of the mean. The critical micelle concentration was qualitatively determined from the visual inflection point in the curves. 

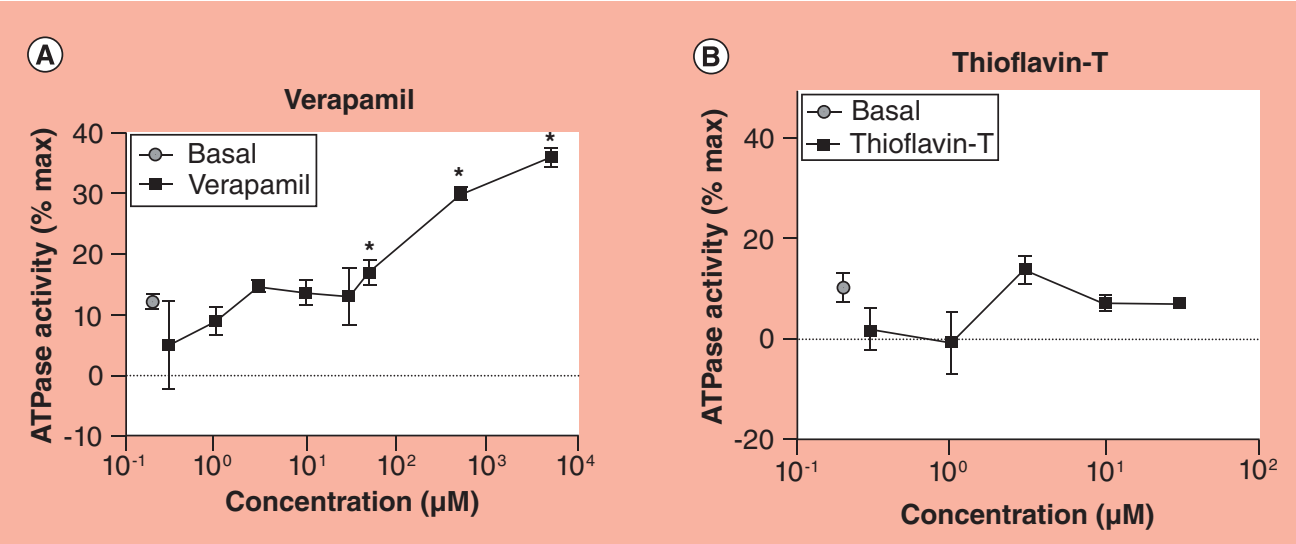

(C)

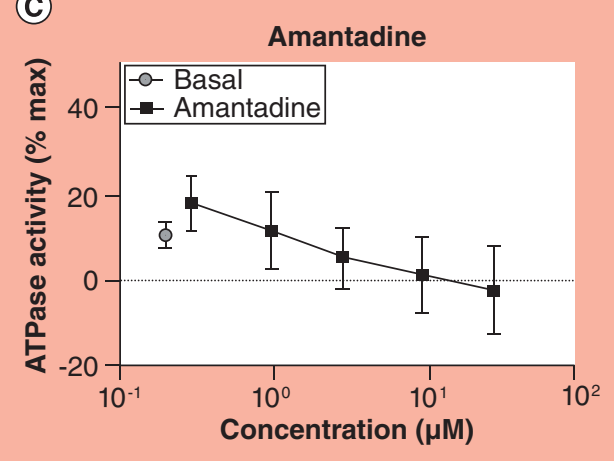

Figure 5. Modulation of P-glycoprotein ATPase activity by various control compounds at several concentrations. (A) Verapamil, (B) thioflavin-T and (C) amantadine. The basal P-gp ATPase activity data point is represented separately from the test compound concentration data points. Experiments were repeated three-times, and the mean ratio \pm standard error of the mean was presented.

$\mathrm{p}<0.005$, one-way ANOVA analysis (*treatment vs basal).

- Octanol/water distribution coefficient (Log P) is not greater than 5 .

- Hydrogen-bond donors (HBDs) are less than 5.

- Hydrogen-bond acceptors (HBAs) are less than 10.

- Number of rotatable bonds is less than 10.

The BBB also contains membrane efflux transporters such as the P-gp efflux pump, which expels many substances attempting to enter the brain with broad specificity. In order to approximate how a drug will interact with P-gp, the measurement of its effect on the rate of P-gp ATP hydrolysis can be determined. P-gp has a basal ATPase activity that may be affected by TCs as follows:

- Compounds that do not interact with P-gp will not change the basal ATPase activity.

- Compounds that may be a substrate for P-gp would stimulate the basal ATPase activity.

- Compounds that may inhibit the induced ATPase activity of another substrate would be competitive inhibitors of P-gp.

- Compounds that inhibit P-gp noncompetitively would inhibit both the basal and stimulated ATPase activity.

For a drug to move into the brain, it has to have a minimal interaction with P-gp. Verapamil - which was used as a positive control in this project - induces the basal P-gp activity [44], while it also can be a P-gp competitive inhibitor by preventing another P-gp substrate from binding to the transporter. Amantadine is used as a no-effect control [45].

Passive diffusion into the brain through the BBB is a standard and straightforward way to enter the brain. The tight junctions of the $\mathrm{BBB}$ are considered as the primary mechanism for inhibiting the passage of water soluble therapeutic drugs for different CNS diseases [42]. The BBB allows compounds with greater lipophilic properties 


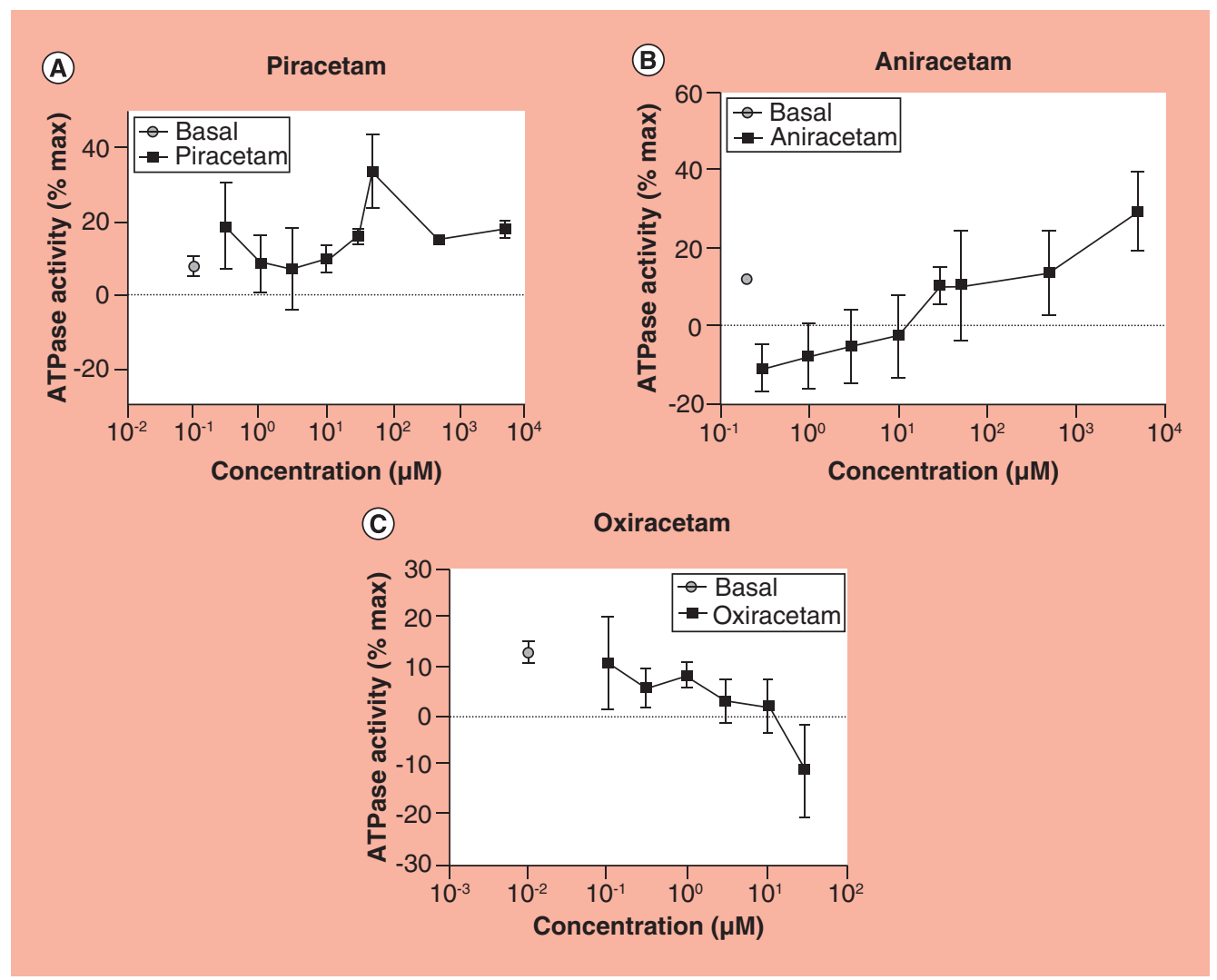

Figure 6. Modulation of P-glycoprotein ATPase activity by various racetam-related nootropic compounds at several concentrations. (A) Piracetam, (B) aniracetam and (C) oxiracetam. The basal P-gp ATPase activity data point is represented separately from the test compound concentration data points. Experiments were repeated three-times, and the mean ratio \pm standard error of the mean was presented.

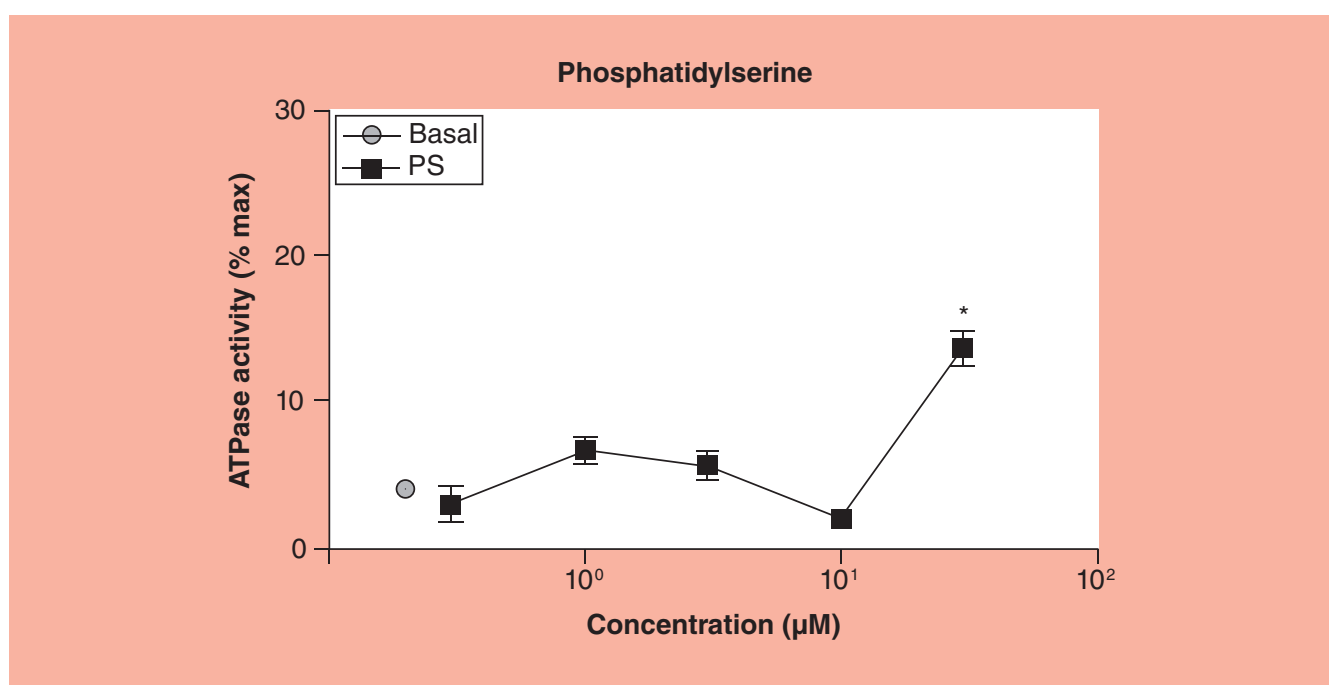

Figure 7. Modulation of P-glycoprotein ATPase activity by neuro-nutrient supplement phosphatidylserine. The basal P-gp ATPase activity data point is represented separately from the test compound concentration data points.

Experiments were repeated three-times, and the mean ratio \pm standard error of the mean was presented. ${ }^{*} \mathrm{p}<0.005$, one-way ANOVA analysis (* treatment vs basal).

PS: Phosphatidylserine. 


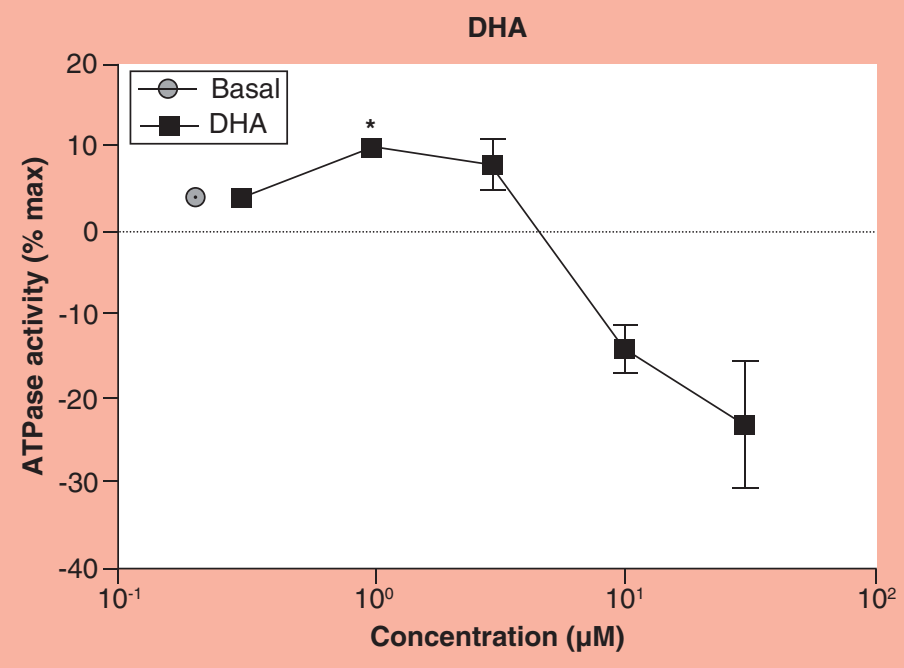

Figure 8. Modulation of P-glycoprotein ATPase activity by neuro-nutrient supplement docosahexaenoic acid. The basal P-gp ATPase activity data point is represented separately from the test compound concentration data points. Experiments were repeated three-times, and the mean ratio \pm standard error of the mean was presented. ${ }^{*} \mathrm{p}<0.005$, one-way ANOVA analysis (*treatment vs basal).

DHA: Docosahexaenoic acid.

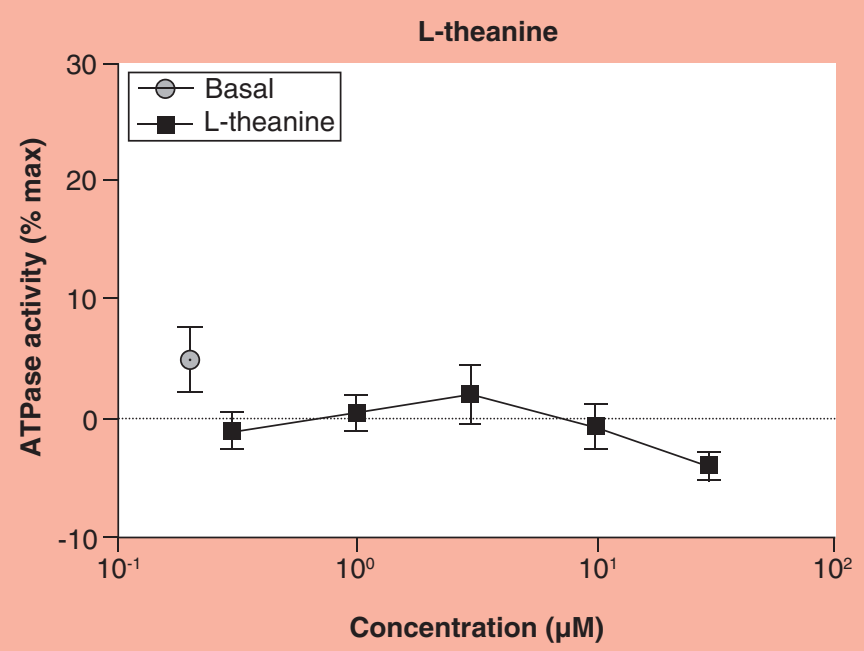

Figure 9. Modulation of P-glycoprotein ATPase activity by L-theanine. The basal P-gp ATPase activity data point is represented separately from the test compound concentration data points. Experiments were repeated three-times, and the mean ratio \pm standard error of the mean was presented.

to cross the BBB by passive transcellular diffusion and the hydrogen bonding properties of drugs can significantly influence their CNS uptake profiles [43,44]. Polar molecules are poor CNS-active drugs unless they can undergo active transport across the CNS. Size, ionization properties and molecular flexibility are other factors observed to influence the transport of an organic compound across the BBB [40].

The comparison of in silico and in vitro data predicts that some, but not all, of the selected nootropics may cross the BBB in vivo. The focus on this project was limited to the passive transcellular diffusion and the interaction with active efflux transporter P-gp for selected nootropic compounds; we did not consider paracellular permeability or 


\begin{tabular}{|c|c|c|c|c|}
\hline \multirow[t]{2}{*}{ Compound } & \multicolumn{2}{|c|}{ In silico data } & \multicolumn{2}{|c|}{ In vitro data } \\
\hline & BBB category & P-gp category & P-gp ATPase activity & CMC value \\
\hline Oxiracetam & No & No & No & High \\
\hline Aniracetam & Yes & No & No & High \\
\hline L-Theanine & No & No & No & Low \\
\hline Phosphatidylserine & No & Yes & Yes & Low \\
\hline Thioflavin-T & Yes & No & No & High \\
\hline Verapamil & No & Yes & Yes & Low \\
\hline Amantadine & Yes & No & No & High \\
\hline
\end{tabular}

other BBB transporter proteins such as breast cancer resistance protein (BCRP), organic anion transporters (OATs) and organic cation transporters (OCTs) [46]. The control compounds verapamil, amantadine and CHAPS all behaved as expected in the in silico and high throughput in vitro screens, but thioflavin-T shows unexpectedly high hydrophilic behavior by having a high CMC value. The data for DHA and aniracetam suggest that these two drugs may cross the BBB despite the P-gp ATPase activity of DHA at $1 \mu \mathrm{M}$ and the high CMC value for aniracetam. PS may be subjected to the P-gp efflux pump, as per both in silico and in vitro data. Data also suggest that piracetam, L-theanine and oxiracetam have a less ability for BBB penetration, as they show more hydrophilic behavior. All in silico and in vitro data from this study are summarized qualitatively in Table 4. The discrepancies in some of the results may be due to the low resolution of most high throughput screening (HTS) techniques. The experiments in this project did not examine the ability of the compounds to affect stimulated P-gp ATPase, nor did they examine specific inhibition of P-gp. Discrepancies with the computational data may be the result of compounds interfering with or reducing the assay's luminescent signal in a manner that is independent of P-gp ATPase stimulation. HTS techniques are most appropriately employed to rank-order or 'bin' compounds by activity. Further supportive in vitro and in vivo studies are needed to support the outcomes of this project.

In conclusion, the physicochemical properties and pharmacodynamic characteristics of nootropic drugs and supplements may be critical elements for determining their safety and efficacy. Comparison of this project's computational and biochemical results suggest that the nootropics with high lipophilicity (high log $\mathrm{P}$, low $\mathrm{H}$ bonding, low $\mathrm{CMC}$ ) and inactivity versus $\mathrm{P}$-gp are best suited to penetrate the BBB in vivo. Accordingly, DHA and aniracetam possess indicators of excellent-to-good BBB permeability in these experiments; whereas, the data are less conclusive for piracetam, L-theanine and oxiracetam. PS may have poor BBB permeability. Therefore, computational and empirical HTS-lead optimization assay technologies may be useful tools for rapid rank-order of drug-like parameters for nootropic supplements in drug discovery. The results of this project cast doubts on the efficacy of some nootropic supplements due to their poor pharmacokinetic properties.

\section{Summary points}

- Computational, physicochemical and biochemical data were compared with the estimated ability of selected nootropic drugs and supplements to penetrate the brain.

- Docosahexaenoic acid (DHA) and aniracetam appear to possess good passive brain permeability properties and avoid efflux by P-gp.

- Computational and empirical high throughput screening (HTS) lead optimization assay technologies may be useful tools for rapid rank-order of drug-like parameters for nootropic supplements in drug discovery. 
Ethical conduct of conduct

The authors state that they have obtained appropriate institutional review board approval or have followed the principles outlined in the Declaration of Helsinki for all human or animal experimental investigations. In addition, for investigations involving human subjects, informed consent has been obtained from the participants involved.

\section{Open access}

This work is licensed under the Attribution-NonCommercial-NoDerivatives 4.0 Unported License. To view a copy of this license, visit http://creativecommons.org/licenses/by-nc-nd/4.0/

\section{References}

Papers of special note have been highlighted as: $\bullet$ of interest

1. Amadio M, Govoni S, Alkon DL, Pascale A. Emerging targets for the pharmacology of learning and memory. Pharmacol. Res. 50(2), 111-122 (2004).

2. Lanni C, Lenzken SC, Pascale A et al. Cognition enhancers between treating and doping the mind. Pharmacol. Res. 57(3), 196-213 (2008).

3. Sharma A, Madaan V, Petty FD. Exercise for mental health. Prim. Care Companion J. Clin. Psychiatry 8(2), 106 (2006).

4. Giurgea C. The 'nootropic' approach to the pharmacology of the integrative activity of the brain. Cond. Reflex 8(2), 108-115 (1973).

5. Malykh AG, Sadaie MR. Piracetam and piracetam-like drugs: from basic science to novel clinical applications to CNS disorders. Drugs 70(3), 287-312 (2010).

- Summarizes piracetam-like drugs.

6. Chinthapalli K. The billion dollar business of being smart. BMJ 351(8025), h4829 (2015).

7. Guo Y, Au WC, Shakoury-Elizeh M et al. Phosphatidylserine is involved in the ferrichrome-induced plasma membrane trafficking of Arn1 in Saccharomyces cerevisiae. J. Biol. Chem. 285(50), 39564-39573 (2010).

8. Albertson TE, Chenoweth JA, Colby DK, Sutter ME. The changing drug culture: use and misuse of appearance- and performance-enhancing drugs. FP Essent. 441, 30-43 (2016).

9. Bostrom N, Sandberg A. Cognitive enhancement: methods, ethics, regulatory challenges. Sci. Eng. Ethics 15(3), 311-341 (2009).

10. O’Hara R, Derouesne C, Fountoulakis KN, Yesavage JA. Therapeutic approaches to age-associated neurocognitive disorders. Dialogues Clin. Neurosci. 3(3), 191-213 (2001).

11. Shorvon S. Pyrrolidone derivatives. Lancet 358(9296), 1885-1892 (2001).

12. Copani A, Genazzani AA, Aleppo G et al. Nootropic drugs positively modulate alpha-amino-3-hydroxy-5-methyl-4-isoxazolepropionic acid-sensitive glutamate receptors in neuronal cultures. J. Neurochem. 58(4), 1199-1204 (1992).

13. Lorier AR, Funk GD, Greer JJ. Opiate-induced suppression of rat hypoglossal motoneuron activity and its reversal by ampakine therapy. PLoS ONE 5(1), e8766 (2010).

14. Waegemans T, Wilsher CR, Danniau A, Ferris SH, Kurz A, Winblad B. Clinical efficacy of piracetam in cognitive impairment: a meta-analysis. Dement. Geriatr. Cogn. Disord. 13(4), 217-224 (2002).

15. Holinski S, Claus B, Alaaraj N et al. Cerebroprotective effect of piracetam in patients undergoing coronary bypass burgery. Med. Sci. Monit. 14(11), PI53-PI57 (2008).

16. Pranzatelli MR. The pharmacology of antimyoclonic drugs. Clin. Neurosci. 3(4), 246-252 (1995).

17. Genton P, Guerrini R, Remy C. Piracetam in the treatment of cortical myoclonus. Pharmacopsychiatry 32(Suppl. 1), 49-53 (1999).

18. Somnier FE, Ostergaard MS, Boysen G, Bruhn P, Mikkelsen BO. Aniracetam tested in chronic psychosyndrome after long-term exposure to organic solvents. a randomized, double-blind, placebo-controlled cross-over study with neuropsychological tests. Psychopharmacology 101(1), 43-46 (1990).

19. Perucca E, Albrici A, Gatti G, Spalluto R, Visconti M, Crema A. Pharmacokinetics of oxiracetam following intravenous and oral administration in healthy volunteers. Eur. J. Drug Metab. Pharmacokinet. 9(3), 267-274 (1984).

20. McLean A Jr, Cardenas DD, Burgess D, Gamzu E. Placebo-controlled study of pramiracetam in young males with memory and cognitive problems resulting from head injury and anoxia. Brain Inj. 5(4), 375-380 (1991).

21. Gomez-Pinilla F. Brain foods: the effects of nutrients on brain function. Nat. Rev. Neurosci. 9(7), 568-578 (2008).

\section{- Summarizes neuro-nutrients.}

22. Calderini G, Aporti F, Bellini F et al. Pharmacological effect of phosphatidylserine on age-dependent memory dysfunction. Ann. $N Y$ Acad. Sci. 444, 504-506 (1985).

23. Graham HN. Green tea composition, consumption, and polyphenol chemistry. Prev. Med. 21(3), 334-350 (1992).

24. Cole GM, Ma QL, Frautschy SA. Dietary fatty acids and the aging brain. Nutr. Rev. 68(Suppl. 2), S102-S111 (2010). 
25. Salem N Jr., Litman B, Kim HY, Gawrisch K. Mechanisms of action of docosahexaenoic acid in the nervous system. Lipids 36(9), 945-959 (2001).

26. Cole GM, Frautschy SA. DHA may prevent age-related dementia. J. Nutr. 140(4), 869-874 (2010).

27. Guesnet P, Alessandri JM. Docosahexaenoic acid (DHA) and the developing central nervous system (CNS) - Implications for dietary recommendations. Biochimie 93(1), 7-12 (2011).

28. Hossain MS, Hashimoto M, Gamoh S, Masumura S. Antioxidative effects of docosahexaenoic acid in the cerebrum versus cerebellum and brainstem of aged hypercholesterolemic rats. J. Neurochem. 72(3), 1133-1138 (1999).

29. van de Rest O, Geleijnse JM, Kok FJ et al. Effect of fish oil on cognitive performance in older subjects: a randomized, controlled trial. Neurology 71(6), 430-438 (2008).

30. Stough C, Downey L, Silber B et al. The effects of 90-day supplementation with the omega-3 essential fatty acid docosahexaenoic acid (DHA) on cognitive function and visual acuity in a healthy aging population. Neurobiol. Aging 33(4), 824.e1-824.e3 (2012).

31. Marino G, Kroemer G. Mechanisms of apoptotic phosphatidylserine exposure. Cell Res. 23(11), 1247-1248 (2013).

32. Cenacchi T, Bertoldin T, Farina C, Fiori MG, Crepaldi G. Cognitive decline in the elderly: a double-blind, placebo-controlled multicenter study on efficacy of phosphatidylserine administration. Aging 5(2), 123-133 (1993).

33. Kato-Kataoka A, Sakai M, Ebina R, Nonaka C, Asano T, Miyamori T. Soybean-derived phosphatidylserine improves memory function of the elderly Japanese subjects with memory complaints. J. Clin. Biochem. Nutr. 47(3), 246-255 (2010).

34. Suzuki J, Umeda M, Sims PJ, Nagata S. Calcium-dependent phospholipid scrambling by TMEM16F. Nature 468(7325), 834-838 (2010).

35. Nathan PJ, Lu K, Gray M, Oliver C. The neuropharmacology of L-theanine(N-ethyl-L-glutamine): a possible neuroprotective and cognitive enhancing agent. J. Herb. Pharmacother. 6(2), 21-30 (2006).

36. Cho HS, Kim S, Lee SY, Park JA, Kim SJ, Chun HS. Protective effect of the green tea component, L-theanine on environmental toxins-induced neuronal cell death. Neurotoxicology 29(4), 656-662 (2008).

37. Seelig A, Gottschlich R, Devant RM. A method to determine the ability of drugs to diffuse through the blood-brain barrier. Proc. Natl Acad. Sci. USA 91(1), 68-72 (1994).

- Highlights use of critical micelle concentration (CMC) and Wilhelmy method to determine passive permeability of drugs.

38. Chattopadhyay A, London E. Fluorometric determination of critical micelle concentration avoiding interference from detergent charge. Anal. Biochem. 139(2), 408-412 (1984).

39. Ma D, Cali JJ. Identify P-glycoprotein substrates and inhibitors with the rapid HTS Pgp-Glo assay system. Promega Notes 96, 11-14 (2007).

- Highlights P-gp ATPase assay.

40. Edwards RH. Drug delivery via the blood-brain barrier. Nat. Neurosci. 4(3), 221-222 (2001).

41. Pajouhesh H, Lenz GR. Medicinal chemical properties of successful central nervous system drugs. NeuroRx 2(4), 541-553 (2005).

42. Lohmann C, Huwel S, Galla HJ. Predicting blood-brain barrier permeability of drugs: evaluation of different in vitro assays. J. Drug Target. 10(4), 263-276 (2002).

43. Zhang J, Higashi K, Limwikrant W, Moribe K, Yamamoto K. Molecular-level characterization of probucol nanocrystal in water by in situ solid-state NMR spectroscopy. Int. J. Pharm. 423(2), 571-576 (2012).

44. Leeson P. Drug discovery: chemical beauty contest. Nature 481(7382), 455-456 (2012).

45. Suzuki T, Fukami T, Tomono K. Possible involvement of cationic-drug sensitive transport systems in the blood-to-brain influx and brain-to-blood efflux of amantadine across the blood-brain barrier. Biopharm. Drug Dispos. 36(2), 126-137 (2015).

46. Uchida Y, Ohtsuki S, Katsukura Y et al. Quantitative targeted absolute proteomics of human blood-brain barrier transporters and receptors. J. Neurochem. 117(2), 333-345 (2011). 